\title{
Synergistic Environmental Degradation of Glass Reinforced Polymer Composites
}

\author{
Tianyi Lu, Euripides Solis-Ramos, Yun-Bo Yi and Maciej Kumosa
}

\author{
National Science Foundation Industry/University Cooperative Research Center \\ for Novel High Voltage/Temperature Materials and Structures \\ University of Denver, Clarence M. Knudson Hall 200, \\ 2390 S. York St. Denver CO 80210, USA
}

\begin{abstract}
Synergistic effects involved in the environmental degradation of Glass Reinforced Polymer (GRP) composites were examined in this research. Six GRPs based on E-glass and ECR-glass fibers with four different polymer resins were exposed either individually or in combination to ultraviolet (UV) radiation, water condensation and elevated temperature for approximately 1000 hours. The composites were monitored for weight changes as a function of time and their surfaces were examined after the tests using optical and scanning electron microscopes. A new model of synergistic aging of polymers under UV and water contestation was also proposed. It has been shown that the selected aging conditions created noticeable synergistic effects causing extensive erosion of the polymer matrices of the tested composites which appeared to be much stronger under the combined actions than under individual exposures. The differences in the aging rates under the individual and combined situations were adequately explained using the newly proposed model. It is suggested that the surface erosion of polymer matrices of the GRPs by combined UV and water condensation creates the most effective condition for small polymer particles formed by UV to be subsequently removed by water condensation exposing fresh still undamaged surfaces to further UV degradation. It has also been shown that depending on the size of the particles different particle removal mechanisms occur on polymer surfaces eroded by a cyclic exposure to UV and water condensation.
\end{abstract}

KEY WORDS: Glass Reinforced Polymers, environmental degradation, synergistic effects ultraviolet degradation, condensation degradation. 


\section{Introduction}

Numerous studies have focused on the environmental degradations of Polymer Matrix Composites (PMCs) in general, and more specifically, aging of Glass Reinforced Polymer (GRP) composites caused by a large variety of aggressive environments. Some of the most recent studies can be found in [1-6]. Very few of them, however, have dealt with the synergistic effects of aging of polymers and GRPs under combinations of several aging conditions [6,7 and 8]. Since this work is concerned with synergistic aging of a group of commercially available GRPs under combined UV radiation, moisture, temperature and time, an emphasis is made here to evaluate the research progress made so far in this very important area of polymer and polymer based composite science.

The UV components of sunlight which reach the ground are in the range of 280-400nm [28]. The energy of ground reaching UV photons is comparable to the dissociation energies of polymer covalent bonds [9-11] resulting in a loss of surface gloss, surface discoloration, chalking, flaking of surface resin, pitting, microcracking, and a severe loss of resin [7-13] in GRPs. The damaging effect of water or moisture on polymer composites, on the other hand, is not as harsh as degradation just by UV radiation even at elevated temperatures [7]. However, moisture diffusion into polymer matrix/fiber interfaces can damage the interfaces by micro-cracking [14, 15] especially at elevated temperatures. In addition, hydrolysis of chemical bonds may lead to permanent chemical degradation $[1,15,16]$ and moisture induced swelling [1, 14, 17] of polymers and their composites.

The combined action of both UV and water on polymers and PMCs as a function of time and temperature could be even more severe than the individual effects. Therefore, this topic has been addressed here both experimentally and numerically. It should be stated that the objective of this work was not to rank a group of GRP composites for their resistance to the above aging conditions, but to investigate the fundamental aspects of the combined UV/water aging processes applicable to any GRP, and more generally, to any PMC system. 


\section{Experimental Procedures}

2.1. Tested composites and specimen preparation

The composites tested in this research are listed in Table 1. The exact chemical compositions of the composites were not released for the purpose of this study. Also, the matrix of composite $\mathrm{F}$ is proprietary information.

Table 1: Tested GRPs

\begin{tabular}{c|c|c|c}
\hline Label & Matrix & Glass fiber & $\begin{array}{c}\text { Weight fraction } \\
(\%)\end{array}$ \\
\hline A & Vinyl ester Epoxy & ECR-Glass 1 & 75 \\
\hline B & Vinyl ester Epoxy & E-Glass & 75 \\
\hline C & Vinyl ester Epoxy & ECR-Glass 2 & 80 \\
\hline D & Polyester & ECR-Glass 2 & 80 \\
\hline E & Epoxy & ECR-Glass 2 & 80 \\
\hline F & Proprietary & ECR-Glass 2 & 80 \\
\hline
\end{tabular}

Two groups of unidirectional GRP composites were investigated. In the first group, three composites (A, B and C) with E-glass, ECR-1 glass and ECR-2 glass fibers in a vinyl ester epoxy blend matrix were tested to evaluate the fiber effect on composites' degradation. In the second group, one type of fiber, ECR-2 glass, was considered with four different polymers, namely polyester, vinyl ester, epoxy and a proprietary resin to determine the matrix effect on the degradation processes in composites C, D, E and F. The fibers came from three different glass manufacturers and the composites were supplied by MacLean Power Systems.

$1.3 \mathrm{~m}$ long, $6 \mathrm{~mm}$ thick and $50 \mathrm{~mm}$ wide composite plates were made by pultrusion by an outside manufacturer. The weight fractions of fibers in the six systems were very similar (Table 1). The composites were dry cut before testing into $80 \mathrm{~mm}$ long specimens with special care to ensure minimum damage during cutting. The cut edges of the samples were sealed with a thin layer of a room temperature vulcanization silicone. Subsequently, the specimens were heat-treated for $72 \mathrm{~h}$ in an oven at $80{ }^{\circ} \mathrm{C}$. The other as supplied surfaces of the samples were smooth and glossy before the tests. 


\subsection{Environmental testing and analysis}

A Q-LABQUV/SPRAY/RP chamber was used for individual and combined UV radiation and water condensation aging tests. The UV radiation tests were carried out for 1000 hours with the UV wavelength ranging from 315 to $400 \mathrm{~nm}$ at $80^{\circ} \mathrm{C}$. An irradiance level of $1.50 \mathrm{~W} / \mathrm{m}^{2}$ at 340 $\mathrm{nm}$ was chosen to more than double the maximum irradiance of natural sunlight at noon. The test temperature was lower than the glass transition temperatures, $\mathrm{Tg}$, of the polymer matrices of the composites, which were higher than $120{ }^{\circ} \mathrm{C}$. The relative humidity $(\mathrm{RH})$ was $3 \pm 2 \%$ and was recorded by a relative humidity meter placed inside the chamber.

In the water condensation tests the specimens were continuously exposed to deionized (DI) water vapor condensation at $60{ }^{\circ} \mathrm{C}$ for 1000 hours with $92 \pm 2 \% \mathrm{RH}$. Water condensation was formed by heating deionized water to vapor on a hot panel inside the chamber. In the cyclic UV radiation and water condensation tests, the composites were exposed to alternating cycles consisting of 16 hours of UV at $80{ }^{\circ} \mathrm{C}$ followed by 8 hours of water condensation at $60{ }^{\circ} \mathrm{C}$ for 1000 hours. The relative humidities were $33 \pm 2 \%$ and $92 \pm 2 \%$, respectively.

For weight measurements the specimens were removed every 96 hours from the chamber for about 60 minutes and weighed with an Ohaus Voyager Electronic Balance (precision $0.01 \mathrm{mg}$ ). The specimens were then randomly repositioned in the chamber to ensure uniform exposure on all surfaces. The percent weight changes as a function of time were calculated using Eq (2.1):

$$
\% \text { weight change }=\frac{\mathrm{w}_{\mathrm{f}}-\mathrm{w}_{\mathrm{i}}}{\mathrm{w}_{\mathrm{i}}} \times 100
$$

where $W_{f}$ and $W_{i}$ are the final and initial dry weights of the samples at room temperature.

Optical and scanning electron microscopes (SEM, JSM $500 \mathrm{LV}$ ) were used to evaluate the surface morphology of the specimens after their exposure. All specimens were prepared and mounted using conventional procedures for SEM observations. Most of the work on the surface deterioration in the composite under the above testing conditions has been reported elsewhere [18]. 


\section{Aging Results and Discussions}

\subsection{Surface erosion}

Surface characteristics of all tested composite specimens after their exposures to UV, water condensation and combined UV/condensation were examined optically and by the SEM. Despite significant differences in their original surface properties such as the amounts of exposed glass fibers, fiber distributions, fiber alignment, fiber diameters, etc. [19], all composites, including A, responded in quite similar fashions to all three test environments with the exception of composite D. Therefore, A was picked as an example to illustrate the aging effects on the composites (Figure 1A). The polyester composite (D) had apparently UV blockers applied to its resin which resulted in less surface damage by UV (Figure 1B). D also had more exposed fibers on the surface (approximately 13\%) in comparison with A (2.8\%). In some cases the exposed fiber areas were as large as 50\% (composite E).
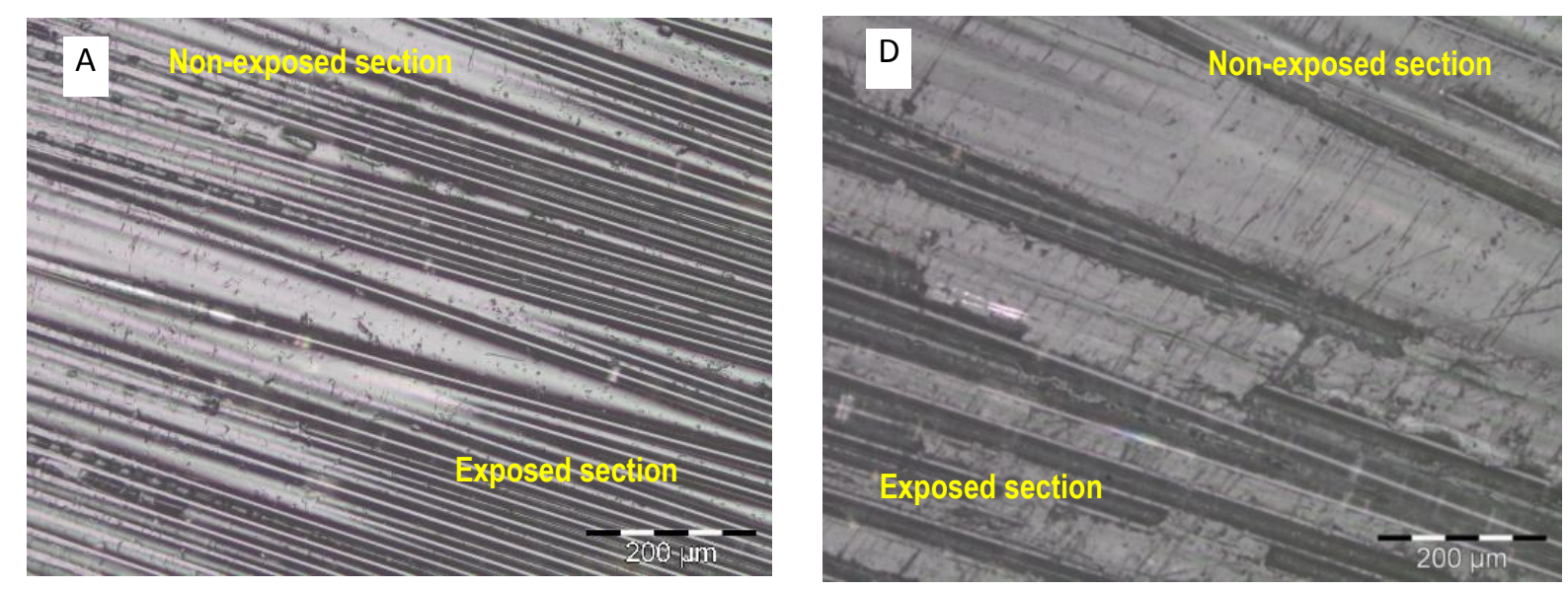

Figure 1. Typical surface characteristics of A and D composites after 1000 hours of exposure to dry hot UV.

Surface damage characteristics under individual UV, water condensation and combined UV and water condensation on Vinyl ester Epoxy ECR-Glass 1 - Composite A are shown in Figure 2 (UV only), Figure 3 (condensation) and Figure 4 (UV/condensation combination). Distinct differences in surface erosion can be observed on the surfaces subjected to the three different aging conditions. 

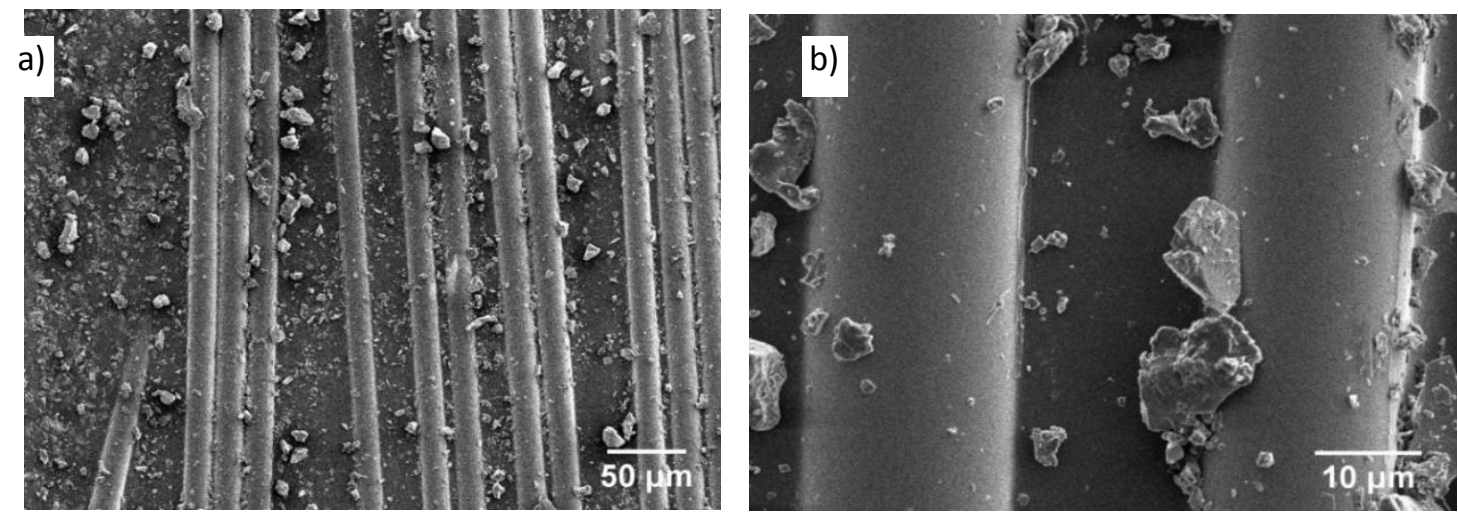

Figure 2. SEM images of exposed surfaces after 1000 hours of UV radiation for Vinyl ester Epoxy ECR-Glass 1 - Composite A; a) low magnification at $\times 300$ and b) high magnification at $\times 1500$.

Figure 2 shows severe matrix degradation with numerous small particles ranging from a micron or smaller to about 20 um formed on the surface by chalking. This phenomenon was caused by the polymer matrix becoming excessively brittle due to an increased crosslinking or the formation of microcracking due to chain scission resulting from photo-oxidation reactions induced by UV radiation [20]. The removed layer of the matrix was more than 20 um deep. The fibers and the fiber/matrix interfaces in all composites were unaffected by UV. Previous research reported that degradation on polymers surfaces by UV is a time dependent effect where the photochemical reactions are restricted to the surface of the polymers within a several micron thick layer [21].
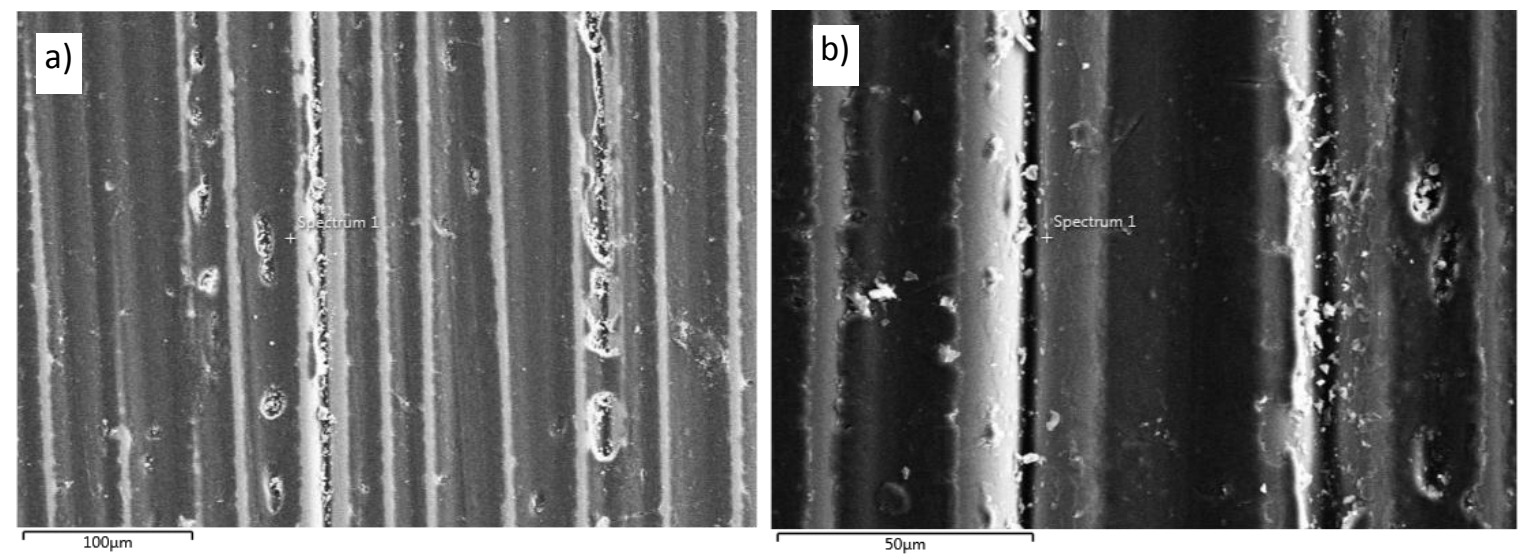

Figure 3. SEM images of exposed surfaces after 1000 hours of condensation for Vinyl ester Epoxy ECR-Glass 1 - Composite A.

a) low magnification at $\times 300$ and $b$ ) high magnification at $\times 1500$ 
The micrographs presented in Figure 3 indicate that the matrix and the interfaces of the composite were degraded by water condensation and the amount of degradation was not uniform across the surface. Overall the amount of damage by water for all composites was much less severe than the damage illustrated in Figure 2 for the UV exposure.
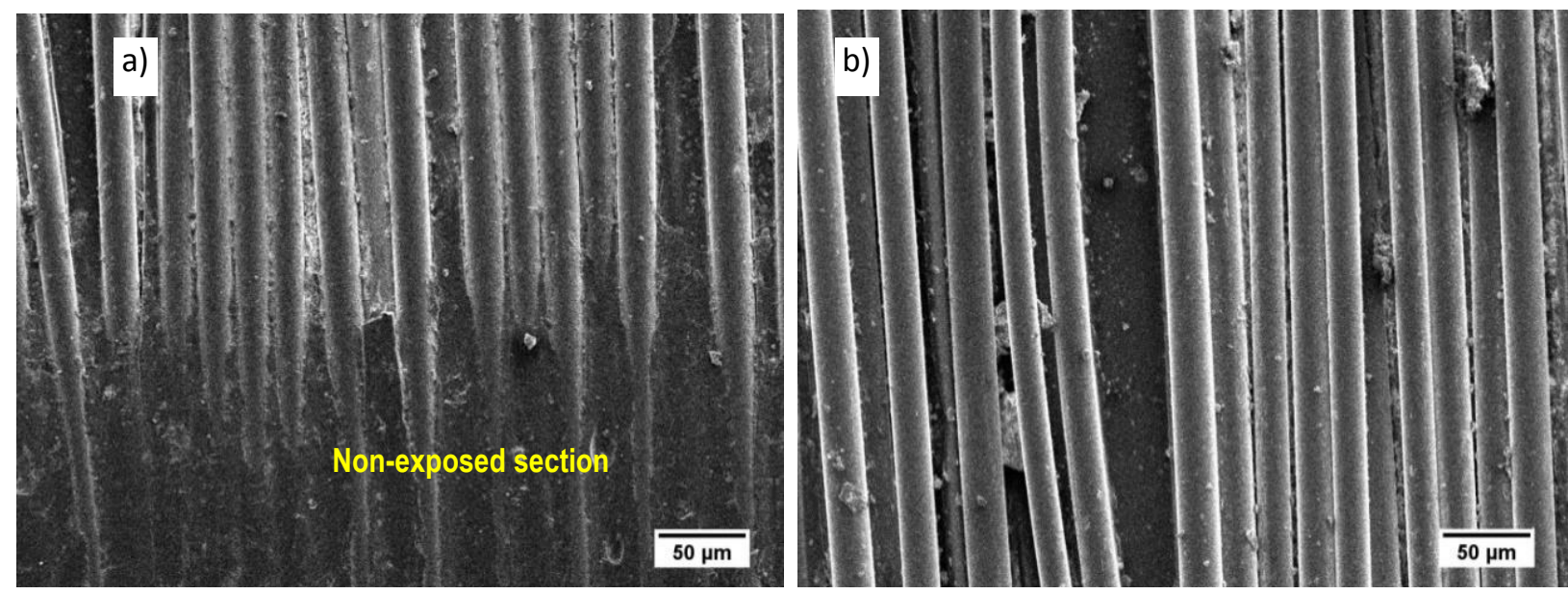

Figure 4. SEM images of exposed surfaces after 1000 hours of UV and condensation for Vinyl ester Epoxy ECR-Composite A.

Cyclic exposure to both UV radiation and condensation resulted in severe degradation that was very different from the other two exposures. The damage consisted of extensive matrix erosion and debonding. As shown in Figure 4, the external polymer layer on the specimen surface was completely removed and the underlying glass fibers were exposed. Most importantly, all samples were almost entirely free from any particles larger than a few microns in diameter.

\subsection{Mass changes from individual and cyclic exposures}

Figure 5 shows weight changes as a function of time for the composites exposed only to UV radiation. The data points for each curve represent averaged measurements obtained from two specimens. It can be observed that the specimens exhibited immediate loss in weight from the very first hours of testing. All GRPs after 1000 hours of exposure lost between 0.03 to $0.056 \%$ 
of their weight. The initial decrease in the weight of the specimens can be attributed to the expulsion of volatiles and residual moisture, which occurred in the first 200 hours of testing. Then, continuous "attack" by UV radiation and oxidation at elevated temperature was the main reason for severe matrix degradation and weight losses.

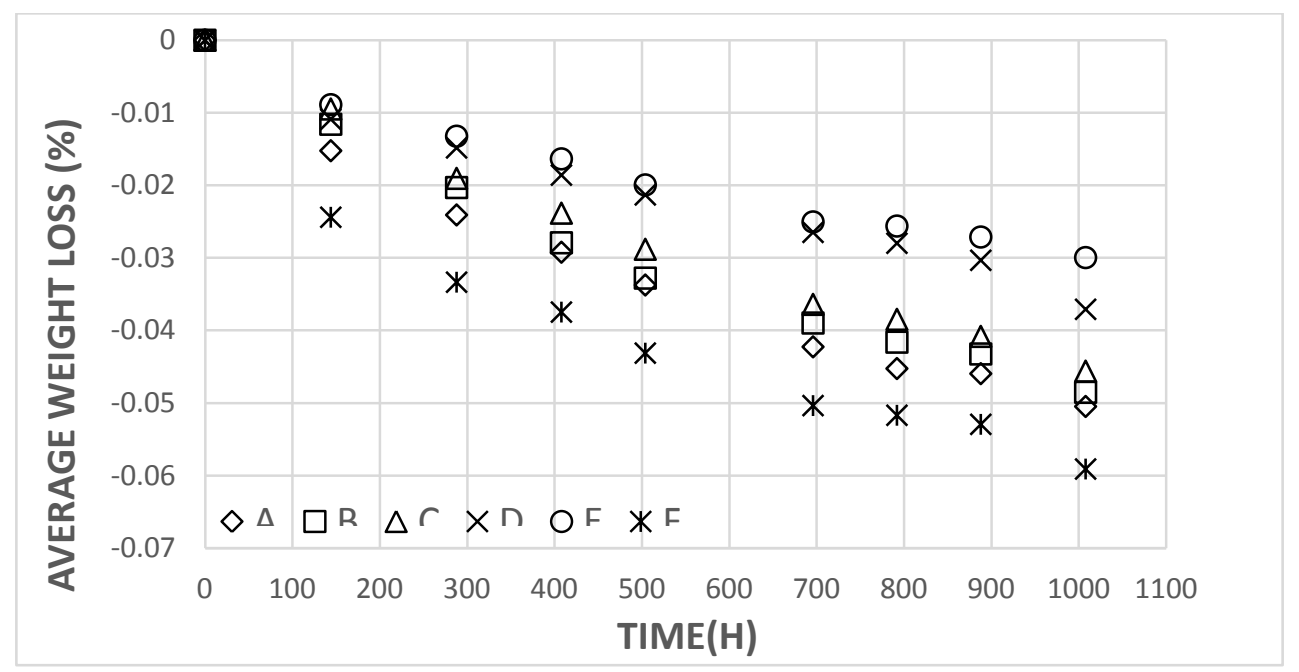

Figure 5. Weight changes for six composites under UV radiation as a function of time.

The effect of water condensation on the weight of the specimens was entirely different in comparison with the UV tests. As shown in Figure 6, all specimens gained weight with time. Again, the data were obtained from two samples for each composite. The specimens gained about $0.04-0.19 \%$ by weight after 1000 hours of exposure depending on their chemical composition without reaching saturation.

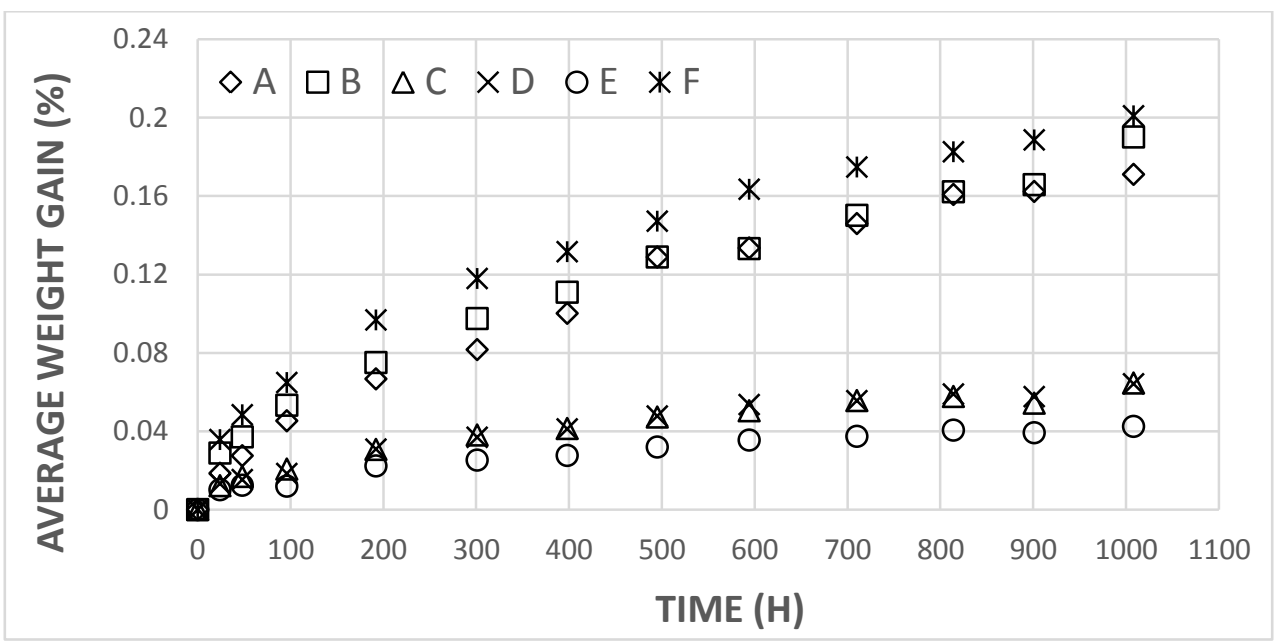

Figure 6. Weight changes for six composites under water condensation as a function of time. 
The combined effect of UV and condensation created another set of weight gain data noticeably different than the individual UV and water condensation exposure. As shown in Figure 7 the specimens started to exhibit a decrease in weight after about 100 hours of exposure after initial small weight gains. The weight losses continued with increasing rates for the remainder of testing. Excluding specimen D, an average of 0.09\% decrease in weight was observed after 1000 hours.

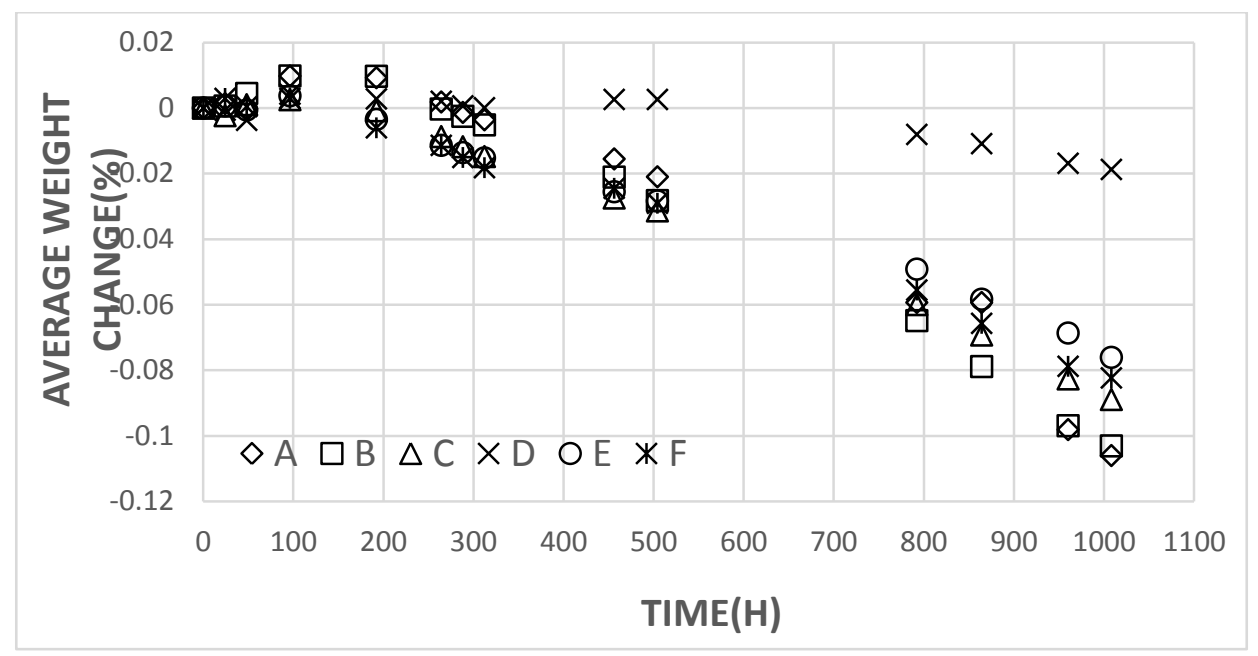

Figure 7.Weight changes for six composites under cyclic UV and water condensation as a function of time.

\section{Synergistic Aging Effects and Their Model}

4.1 Comparison between individual and combined exposures

Despite the fact that the six GRPs investigated in this research were based on three different glass fibers and four different polymer resins with very different surface characteristics, their individual responses to UV, water condensation and the combination of UV and condensation were quite similar. As expected, all of them lost weight under UV exposure (Figure 5). Also, as expected, all of them gained weight when exposed to water condensation (Figure 6). Under the combined action of both UV and condensation, all of them lost weight (Figure 7). The changes in weight within the 1000 hours of testing were not large but significant enough to detect the effect of the three different environments on the composites. If the sums of the weight changes under the individual exposures are compared with the changes in weight under the combined UV/condensation condition, a very interesting effect can be observed immediately (Figure 8). 
The sums of the individual effects on weight changes are positive and much smaller than the changes in weight under the combined environments, which are not only larger but also negative. This clearly demonstrates that the synergistic effects in the aging under $\mathrm{UV} /$ condensation/temperature/time of the six composites are quite strong.

The synergistic effects observed in Figure 8 are even better visible in the averaged weight changes obtained from the six composites and presented in Figure 9. Several important observations can be made here. First, the averages of the individual effects on the weight changes for the six composites are positive and about 50\% smaller than the averages in the weight changes from the cyclic exposures which are negative. Most importantly, the sum vs. the cyclic average of all composite relations with temperature is very similar to the relations for the individual composites. Therefore, it is possible that the observed synergistic effect is a typical feature for any unidirectional GRP composite exposed to the aging conditions of this research.

Second, the cyclic UV and water condensation resulted in the largest weight reductions, and most likely the largest amounts of damage to all six composites in comparison with the other exposures. Third, the effect of UV on the averages under UV only seems to increase with a decelerating rate. On the other hand, the cyclic effect of UV and water condensation appears to accelerate. Finally, the standard deviations of the averages from the UV tests are the smallest whereas the condensation tests resulted in the largest scatters in weight charges. Regarding the scatters, the averaged sums and averaged cyclic data for all composites are much closer to the condensation scatters than the UV scatters. Condensation alone or in combination with UV seems to create a higher level of uncertainties in the response of the composites to aging. Most likely this is caused by a much stronger and less predictable effect of interfaces in the condensation erosion than in the UV degradation, which seems to be more predictable and dominated by the steady erosion of the matrix between the fibers. 


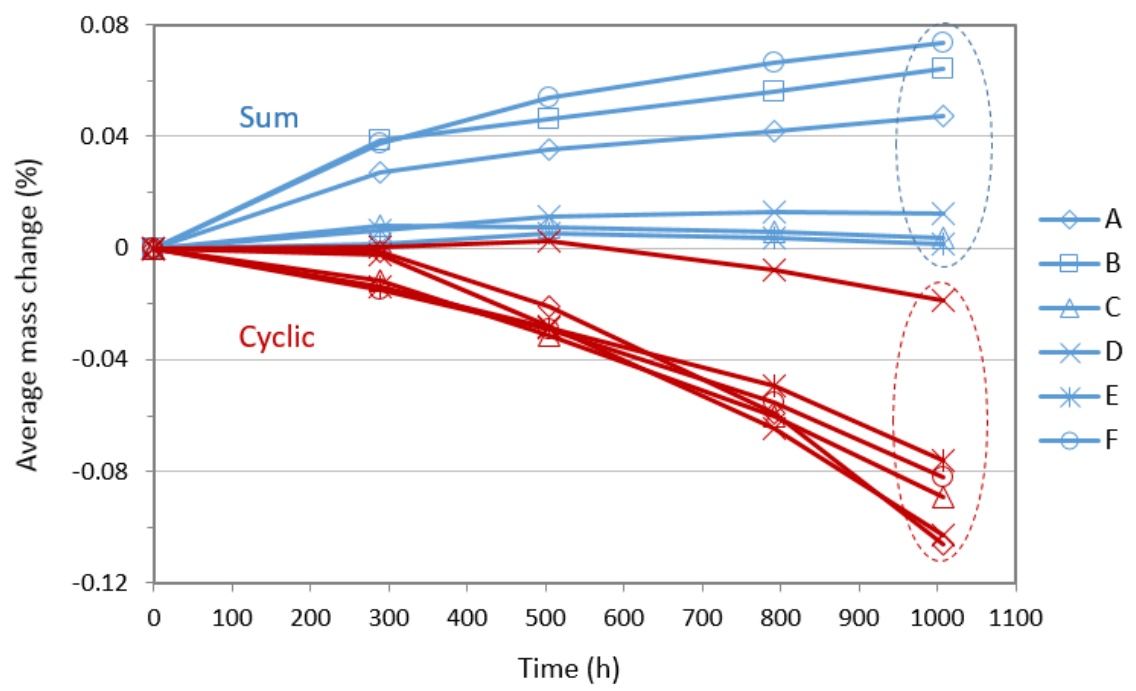

Figure 8. Weight changes for six composites tested under cyclic UV/condensation and the sums of weight changes under individual UV and condensation conditions with time.

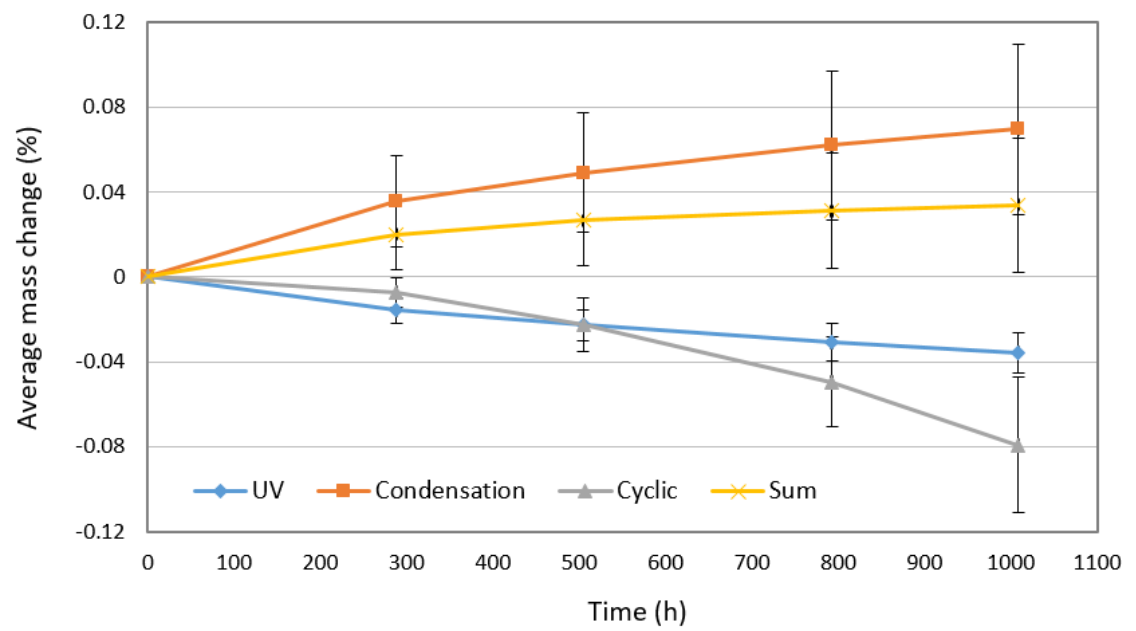

Figure 9. Averaged weight changes with standard deviations for six composites tested under individual UV and water condensation, cyclic UV and condensation, and the averaged sums of weight changes from the individual exposures as a function of time.

Two main mechanisms should be considered to explain why the surfaces of the composites after the cyclic UV/water condensation exposures are severely eroded but almost free from any visible particles (Figure 4). One is based on the chemical degradation of the resins under high energy radiation conditions with oxygen, for example by the formation of hydroperoxides, a significant intermediate $[11,20]$. Those instable intermediates are quite active and result in chain scission and crosslinking after complex reactions. Eventually, these chemical reactions will result in the microcracking and chalking on the exposed surface, which was shown in Figure 2. During the 
condensation cycle, resin hydrolysis and moisture absorption occur [20] but the resin erosion is significantly less severe [7]. In the second step, any soluble and insoluble (particles) products are removed from the ultraviolet radiated surface by slowly moving condensation water. Then a fresh polymer surface is exposed to the following UV cycle and more damage is subsequently created by UV followed by the subsequent cleaning of the surface damage by water in the next condensation cycle.

4.2 Model of synergistic polymer degradation

In support of the above two mechanisms of UV degradation and subsequent removal of UV generated particles by water condensation, a synergistic model of aging has been proposed in this work with the following assumptions:

- Constant air temperature, humidity and UV radiation intensity.

- Contributions from individual wavelengths are independent.

- Only a fraction of the absorbed photons lead to photolytic activity. The photo recovery effect is neglected.

- The initial polymer surface exposed to UV has a sinusoidal shape.

- No water absorption or diffusion occur during condensation exposure.

- Water does not react with polymer.

- No thermal degradation takes place.

- Particles are spherical in shape and the local surface imperfections do not affect the particle removal process.

- UV light is from a line source. When a sinusoidal surface is exposed to radiation, UV intensity is a function of the angle between the surface and the incident light.

- Water flow is laminar and is caused by gravity alone.

\subsubsection{UV radiation model}

Since UV irradiation is the initiator for polymer degradation, an approximate solution is obtained 
for the total effective UV irradiation dosage based on Eq. (4.1) $[9,22,23]$

$$
D_{\text {total }(t)}=\int_{0}^{t} \int_{\lambda_{\min }}^{\lambda_{\max }} E_{o}(\lambda, t)\left(1-e^{-A(\lambda, t)}\right) \phi(\lambda) d \lambda d t
$$

where

- $\lambda$ min: minimum photolytically effective wavelengths $(\mathrm{nm})$

- $\lambda$ max: maximum photolytically effective wavelengths $(\mathrm{nm})$,

- $\mathrm{A}(\lambda, t)$ : the absorbance of the light of wavelength $\lambda$ in the material and at time $t$, (dimensionless),

- Eo $(\lambda, t)$ : incident spectral UV radiation dose to which a polymeric material is exposed to at time $\mathrm{t}\left(\mathrm{J} \mathrm{cm}^{-2}\right)$,

- $t$ : elapsed time (second).

- $D_{\text {total }}(t)$ : total effective dosage $(J)$

- $\Phi(\lambda)$ : quantum yield, which is the number of times a specific event occurs per photon absorbed by the material.

A linear function is then used to establish a relationship between the total irradiation dosage after a certain amount of time, $D_{\text {total }}$, and the amount of UV damage defined by the local damage thickness, $\Gamma,[23]$

$$
\Gamma=a \times D_{t o t}
$$

where $a$ is a factor indicating the efficiency with which the UV degraded material is removed from the surface during UV exposure only.

The intensity of irradiation for any angle of UV light with respect to the surface is determined by

$$
E_{0}=I_{0}(\lambda, \theta, t) \cos (\theta)
$$

where $I_{\mathrm{o}}$ is the intensity of $\mathrm{UV}\left(\mathrm{w} / \mathrm{m}^{2} \cdot \mathrm{s}\right)$, and $\theta$ is the angle between the UV light and the normal direction to the surface. 
The numerical differentiation method was adopted to solve Equations 4.1-4.3 in the simulations of UV degradation of polymer surfaces with various sinusoidal profiles. All physical input data used in the simulations are listed in Table 2. If we assume that (1) all UV generated particles formed on the degraded surface are immediately removed after their formation so a "fresh" surface is subsequently exposed to UV, (2) a parameter " $a$ " is selected, and (3) the incident UV light shines perpendicularly to the horizontal direction, the stages of surface reconfiguration by UV of a standard sinusoidal surface with the arithmetical mean roughness of 0.466 can be observed in Figure 10. For the selected light direction there are the maxima of degradation rates at the peaks and the valleys of the surface where the degradation rates are faster than those at the other locations.

Table 2: Input data for performed UV damage simulations.

\begin{tabular}{|l|l|}
\hline Parameter & Value \\
\hline Wavelength & $340 \mathrm{~nm}$ \\
\hline Radiation intensity, Io & $1.5 \mathrm{~W} / \mathrm{m}^{2}$ \\
\hline Quantum yield $\Phi$ - [24] & $10^{-4}$ \\
\hline Exposure time t & 1000 hours \\
\hline Exposure area & $3.25 \times 10^{-3} \mathrm{~m}^{2}$ \\
\hline $\begin{array}{l}\text { Efficiency of material removal; } \\
\text { arbitrarily chosen }\end{array}$ & 0.1 \\
\hline
\end{tabular}

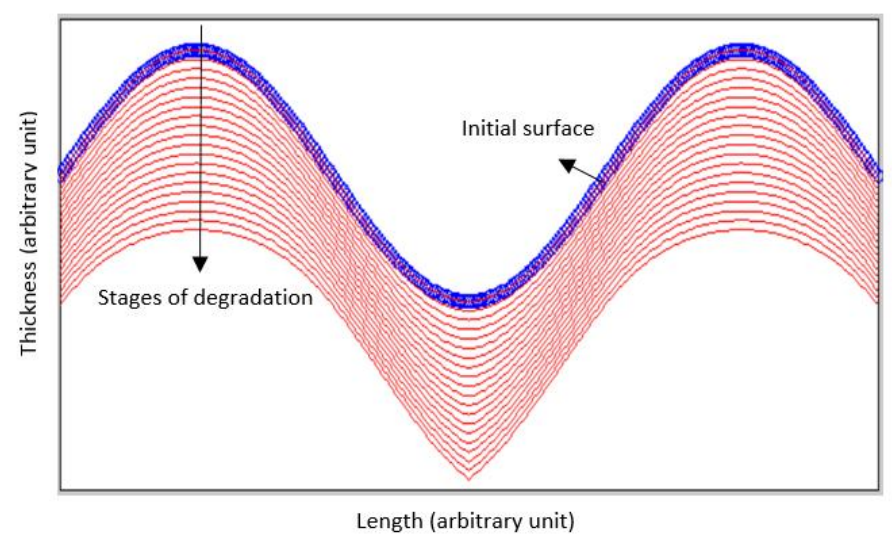

Figure 10. Stages of UV degradation of a sinusoidal surface. 


\subsubsection{Hydrodynamic and particle removal models}

After UV exposure for a certain period of time there will be a number of small particles of different shapes and sizes formed on the polymer surface (see Figure 2). These particles will be subsequently washed away by the drag force of the water flow in the condensation cycle. The drag is caused by the viscous shear stresses exerted on the solid-fluid interface. The simulation results of the water velocity and shear stress distribution on the polymer surface are shown in Figure 11 based on the commercial finite element software COMSOL, using the physical data listed in Table 3 and for the same initial sinusoidal surface as assumed in Figure 10. The open boundary condition was assumed on the water surface. It can be seen in Figure 11 that there are periodic oscillations in the viscous stresses on the polymer surface caused by the initial surface configuration. The decrease in the shear stress in Figure 11b was caused by an increase in the velocity of the water flow from an initial value of $10 \mathrm{~mm} / \mathrm{sec}$ to $340 \mathrm{~mm} / \mathrm{sec}$ at the end of the specimen.

Table 3. Input data used in the hydrodynamic model.

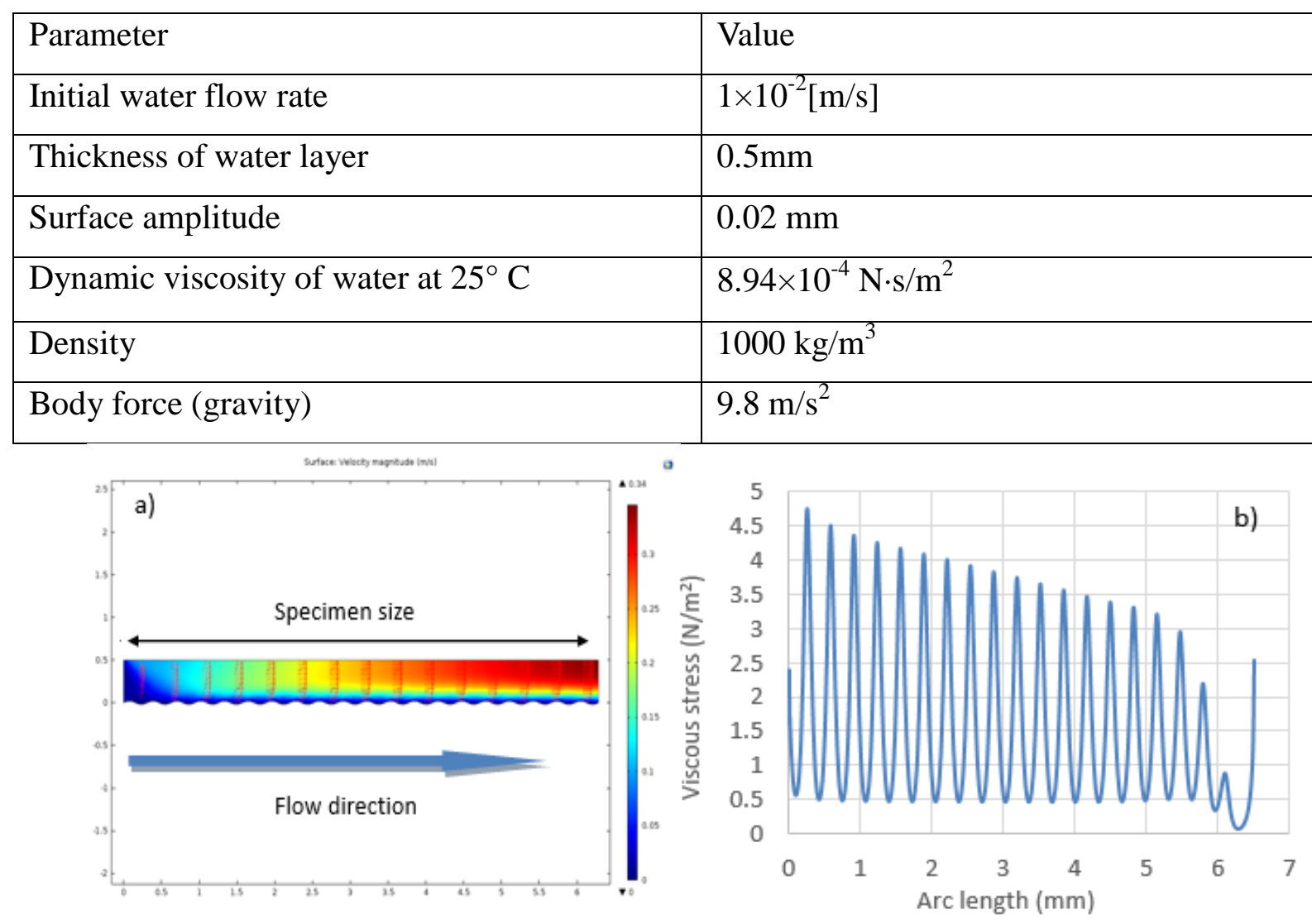

Figure 11. (a) Velocity of water flow; (b) Viscous stress along the surface. 
To determine whether the UV generated particles can be removed by water flow, the drag force has been compared with the Coulomb friction between the particles and the solid surface. For micro-scale particles, the effects of gravity and buoyancy are relatively small and can usually be neglected [25]. As a result, the friction is proportional to the normal stress acting on the surface, which is solely determined by the van der Waals forces. For simplicity, the particles are assumed to have spherical shapes for estimating the van der Waals forces. In addition, three different particle removal mechanisms namely lifting, sliding and rolling have been considered in the model $[25,26,27]$.
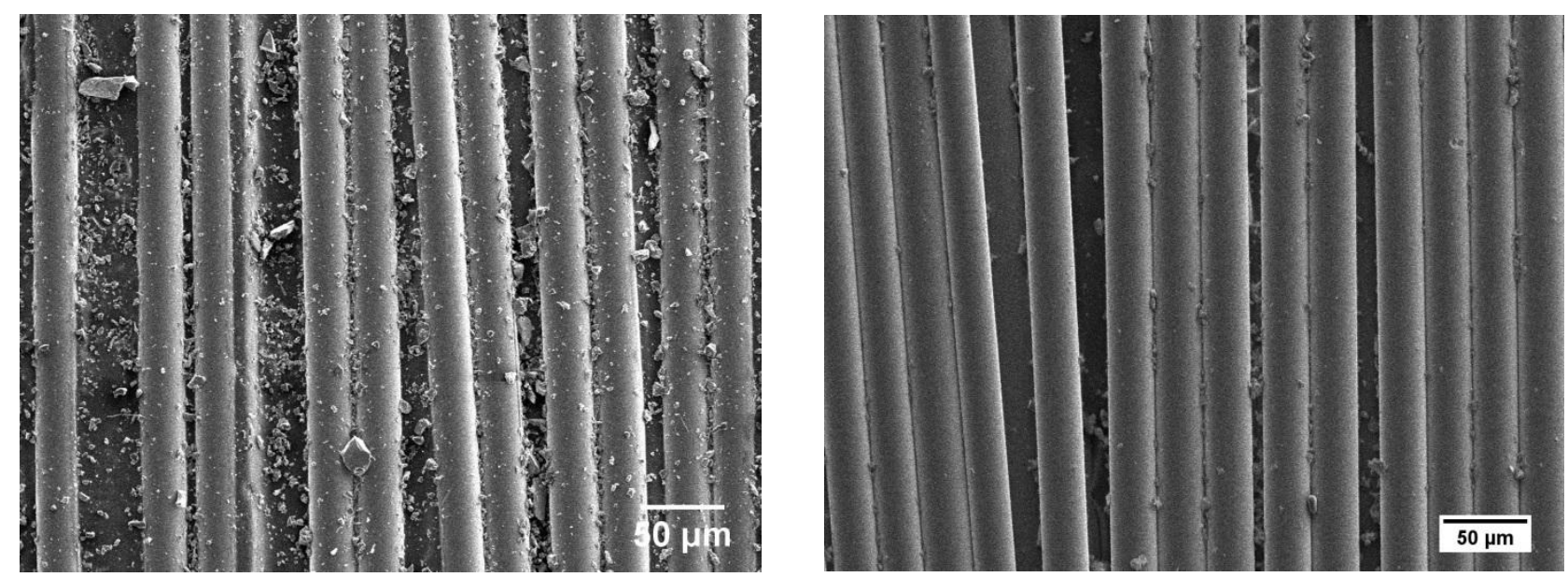

Figure 12. SEM images of composites E after (a) UV radiation and (b) UV \& water condensation.

The Johnson-Kendall-Roberts (JKR) model [26] has been used to calculate the van der Waals forces. By combining an average shear stress of $1.59 \mathrm{~N} / \mathrm{m}^{2}$ from our hydrodynamic model, contact mechanics [26] and particle detachment equations [25,26,27], preliminary results have been obtained showing that particles of diameter larger than $1.3 \mu \mathrm{m}$ can roll, and that the sliding type of motion is possible for particles of diameters $34.2 \mu \mathrm{m}$ and above. Particles larger than $84.6 \mu \mathrm{m}$ will be lifted by water. In other words, particles larger than $1.3 \mathrm{um}$ in diameter will be removed from the surface by three different mechanisms, whereas those smaller than 1.3 um will adhere to the surface.

In order to validate the predictions, more SEM imaging was performed on the ECR 1/epoxy composite (Figure 12) after the combined UV/water condensation exposure. The results showed 
that a slow water flow during condensation removed all larger particles $(2-10 \mu \mathrm{m})$ after UV radiation; however, some smaller particles $(<1 \mu \mathrm{m})$ were still adhered to the surface, as seen in Figure 12b. Similar observations can be made from the surface characteristics presented in Figures 4. After the removal of the larger particles by water condensation, a "fresh" surface of the polymer matrix is exposed to UV again, and the process continues with a slowly increasing rate (Figures 8 and 9).

\section{Conclusions}

It has been shown in this study that commercially available unidirectional GRP composites when exposed to individual and cyclic UV radiation, water condensation and temperature develop significantly different degradation mechanisms with strong synergistic effects. Most importantly the sums of the individual UV and water condensation aging effects on weight changes are positive with weight gains, whereas the weight changes under the cyclic combined conditions are negative with weight losses for all six tested composites. The average weight losses under cyclic conditions for all six composites were found to be about $100 \%$ larger than the weight gains from the simple superposition of the UV and condensation environments for the same composites.

To explain the synergistic effect observed in this work, a new hypothesis of damage initiation and progression in the GRPs subjected to both UV and water condensation was formulated and supported by a new model of synergistic aging. According to our model, under the combined action of UV and water condensation small particles are first formed on the surfaces of polymeric surfaces exposed to UV radiation. The particles are subsequently removed by the slowly moving water caused by the condensation, which exposes fresh undamaged polymer surfaces to the cycle of UV. This creates a much more severe surface erosion than UV alone. Using our still very preliminary model, UV generated polymer particles found on the surfaces of the tested GRPs after UV were evaluated for their response to water cleaning during the condensation cycle. It was determined that most of the particles generated during the combined testing should be removed by water condensation. This prediction was successfully verified by SEM observations of the environmentally aged composites. 


\section{Acknowledgments}

This research was supported jointly by the MacLean Power System Corporation between October 1, 2012 and June 30, 2014, and since June 2014 by the National Science Foundation Industry/University Cooperative Research Center for Novel High Voltage/High Temperature Materials and Structures under \#IIP1362135, and by the National Science Foundation Grant Opportunities for Academic Liaison with Industry program under \#CMMI-123252.

\section{Reference}

1. Yinghui Hu, Xiaochen Li, Augustus W. Lang, Yunpeng Zhang, Steven R. Nutt Water immersion aging of polydicyclopentadiene resin and glass fiber composites Polymer Degradation and Stability,124 (2016), Pages 35-42

2. Joung-Man Park, Pyeong-Su Shin, Zuo-Jia Wang, Dong-Jun Kwon, Jin-Yeong Choi, Sang-II Lee, K. Lawrence DeVries

The change in mechanical and interfacial properties of GF and CF reinforced epoxy compositesafter aging in $\mathrm{NaCl}$ solution

Composites Science and Technology 122 (2016) 59-66

3. Jun Wang, HotaGangaRao, Ruifeng Liang, Ding Zhou, Weiqing Liu and Yuan Fang Durability of glass fiber-reinforced polymer composites under the combined effects of Moisture and sustained loads Journal of Reinforced Plastics and Composites 2015, Vol. 34(21) 1739-1754

4. Zhi Chen, Lifei Zheng, QingpingJin, Xiaoqing Li

Durability study on glass fiber reinforced polymer soil nail via accelerated aging test andlong-term field test

Polymer Composites 2015 1-11

5. Paul Böer, Lisa Holliday, Thomas H.-K. Kang Independent environmental effects on durability of fiber-reinforced polymer wraps in civil applications: A review

Construction and Building Materials 48 (2013) 360-370

6. G. Carra, V. Carvelli

Ageing of pultruded glass fibre reinforced polymer composites exposed to combined environmental agents

Compos. Structure, 108 (2014), pp. 1019-1026 
7. B.G. Kumar, R.P. Singh, T. Nakamura

Degradation of carbon fiber-reinforced epoxy composites by ultraviolet radiation and Condensation

J. Compos Mater, 36 (2002), pp. 2713-2721

8. Dionysis E. Mouzakis $b,{ }^{*}$, Helen Zoga a , Costas Galiotisa,b

Accelerated environmental ageing study of polyester/glass fiber reinforced composites(GFRPCs)

Composites: Part B 39 (2008) 467-475

9. Alexandre François-Heude, Emmanuel Richaud, Eric Desnoux, Xavier Colin

Influence of temperature, UV-light wavelength and intensity on polypropylenePhotothermalOxidation

Polymer Degradation and Stability 100 (2014) 10-20

10. Ranby, B. and Rabek, J.F.

Photodegradation, Photo-Oxidation and Photostabilizationof Polymers, John Wiley and Sons, London.1975

11. George Wypych

Handbook of UV degradation and stabilization

ChemTec Publishing 2011

12. Andrew W Signor, Mark R VanLandingham, Joannie W Chin

Effects of ultraviolet radiation exposure on vinyl ester resins: characterization of chemical,physical and mechanical damage

Polymer Degradation and Stability792003, Pages 359-368

13. Dan Rosu, Liliana Rosu, Constantin N. Cascaval

Effect of ultraviolet radiation on vinyl ester network based on bisphenol A Journal of Photochemistry and Photobiology A: Chemistry 194 (2008) 275-282

14. L. Gautier, B. Mortaigne, V. Bellenger

Interface damage study of hydrothermally aged glass fibre-reinforced polyester composite

Composites Science and Technology 59 (1999) 2329-2337

15. S. Pavlidou, C.D. Papaspyrides

The effect of hygrothermal history on water sorption and interlaminar shear strength of glass/polyester composites with different interfacial strength

Composites: Part A 34 (2003) 1117-1124

16. Mohdlshak ZA, Ariffin A, Senawi R.

Effects of hygrothermal aging and a silane coupling agent on the tensile properties of 
injection molded short glass fiber reinforced poly(butylene terephthalate) composites. EurPolym J 2001;37:1635-47.

17. Carol L. Schutte

Environmental durability of glass-fiber composites

Materials Science and Engineering, R13 (1994) 265-324

18. T. Lu

Degradation of high voltage glass fiber-reinforced polymer matrix composites by aggressive environmental conditions

MS thesis, University of Denver, Denver Colorado, August 2014.

19. E. Solis-Ramos, J. Hoffman and M. Kumosa,

Synergistic effects in stress corrosion cracking of glass reinforced polymer

composites, to be submitted to Polymer Degradation and Stability, March 2016,

20. Marcos A.J. Batista, Raul P. Moraes1, Jayne C.S. Barbosa, Pedro C. Oliveira, Amilton M.

Santos

Effect of the polyester chemical structure on the stability of polyester-melamine coatings when exposed to accelerated weathering

Progress in Organic Coatings 71 (2011) 265-273

21. J K. Fink, High performance polymers, Elsevier, 2008 pp 347-390

22. Brian Hinderliter, Stuart Croll

Monte Carlo approach to estimating the photodegradation of polymer coatings JCT Research, 2 (2005) 483-491

23. Jonathan W. Martin

Quantitative characterization of spectral ultraviolet radiation-induced photodegradation in coating systems exposed in the laboratory and the field.

Progress in Organic Coatings, 23 (1993) 49-70

24. SørenKiil

Model-based analysis of photoinitiated coating degradationunder artificial exposure conditions

J. Coat. Technol. Res., 9 (4) 375-398, 2012

25. Norbert Riefler, Michael Heiland, Anja Waske, Norbert Rabiger, Stefan Odenbach, Udo

Fritsching

Particle deposition and detachment in capillary sphere packings

Chemical Engineering Journal 174 (2011) 93-101

26. M.L. Zoeteweij, J.C.J. van der Donck and R. Versluis 
Particle removal in linear shear flow: model prediction and experimental validation Journal of Adhesion Science and Technology 23 (2009) 899-911

27. G.M. Burdick, N.S. Berman, S.P. Beaudoin

Describing hydrodynamic particle removal from surfaces using the particle Reynolds number

Journal of Nanoparticle Research 3 (2001) 455-467

28. Wiki: UV light https://en.wikipedia.org/wiki/Ultraviolet 